\title{
Artigo Revisão: Atuação dos antioxidantes em refratários contendo carbono
}

\section{(Review Article: Performance of the antioxidants in carbon containing refractories)}

\author{
A. P. Luz, V. C. Pandolfelli \\ Grupo de Engenharia de Microestrutura de Materiais - GEMM \\ Departamento de Engenharia de Materiais - DEMa, Universidade Federal de S. Carlos - UFSCar \\ Rod. Washington Luiz, km 235, C.P. 676, S. Carlos, SP 13565-905 \\ ana_light@uol.com.br,vicpando@power.ufscar.br
}

\begin{abstract}
Resumo
Os materiais refratários contendo carbono têm despertado grande atenção nos últimos anos, uma vez que são amplamente utilizados na indústria siderúrgica em virtude de suas excelentes propriedades. A presença do carbono nos refratários contribui para o aumento da resistência ao choque térmico, da energia de fratura e da resistência ao ataque por escória. Entretanto, a principal desvantagem do seu uso é a sua alta susceptibilidade a oxidação. Com o objetivo de manter o carbono presente na estrutura do material refratário o maior tempo possível, algumas medidas são requeridas para reduzir sua oxidação sob condições operacionais. Desta forma, o uso de substâncias conhecidas como antioxidantes tem sido extensivamente estudado. Os principais antioxidantes, suas características e formas de atuação são abordados nesta revisão. A formação de novos compostos devido à reação entre os antioxidantes com o carbono, nitrogênio, oxigênio e o próprio material refratário pode provocar um preenchimento parcial dos poros e redução da taxa de interdifusão dos gases, havendo assim a prevenção da oxidação do carbono presente no refratário.

Palavras-chave: antioxidantes, refratários contendo carbono.
\end{abstract}

\section{Abstract}

Carbon containing refractories have received great attention over the last years, as they are widely used in the steel making industry due to their better properties, such as: higher thermal shock resistance, fracture energy and slag resistance. However, their main disadvantage is the carbon oxidation. Aiming to keep carbon in the structure of the refractory material some actions are required in order to reduce its oxidation under operational conditions. Therefore, the use of materials known as antioxidants has been extensively studied. The main antioxidants, their characteristics and performance are discussed in the present review article. The formation of new compounds due to the reaction of the antioxidants with carbon, nitrogen, oxygen and the refractory material can induce a pore content decrease and reduction of the interdifusion rate of the gases, thus preventing the oxidation of the carbon in the refractory material.

Keywords: antioxidants, carbon containing refractories.

\section{INTRODUÇÃO}

Concretos refratários contendo carbono vêm sendo amplamente utilizados em indústrias siderúrgicas principalmente em revestimento de canais de corrida de altofornos. O desempenho bem sucedido de tijolos refratários contendo carbono (por exemplo, $\mathrm{MgO}-\mathrm{C}, \mathrm{Al}_{2} \mathrm{O}_{3}-\mathrm{C}$ e $\mathrm{Al}_{2} \mathrm{O}_{3}$ $\mathrm{SiC}-\mathrm{C}$ ) tem despertado o interesse pelo desenvolvimento de concretos refratários que contenham elevados teores deste material [1-4].

A adição de carbono aos refratários é conhecida por contribuir para o aumento da condutividade térmica, da energia de fratura, da resistência ao dano por choque térmico e aumento da resistência ao ataque por escória em função de sua inferior molhabilidade por óxidos fundidos.
Entretanto, a incorporação de carbono tem como principal desvantagem a sua alta susceptibilidade à oxidação em elevadas temperaturas [5].

A conseqüência imediata da oxidação em refratários contendo carbono é a perda de resistência mecânica decorrente do aumento da porosidade, que promove uma redução da resistência a penetração do ar e escória, o que intensifica o processo de deterioração do material refratário. Portanto, os benefícios decorrentes da adição de carbono em refratários depende do controle de sua oxidação sob condições operacionais.

A oxidação direta do carbono com o oxigênio gasoso $\left(\mathrm{O}_{2}\right)$ inicia-se entre 400 e $1200^{\circ} \mathrm{C}$ e $\mathrm{P}_{\mathrm{O} 2} \geq 10^{-4} \mathrm{~atm}$ (equação A). Em temperaturas superiores a $1400^{\circ} \mathrm{C}$ a oxidação indireta torna-se rapidamente predominante e o carbono reage então com o 
óxido sólido ou líquido ou sub-óxidos gasosos presentes no sistema [6-14].

$$
2 \mathrm{C}_{(\mathrm{s})}+\mathrm{O}_{2(\mathrm{~g})} \rightarrow 2 \mathrm{CO}_{(\mathrm{g})}
$$

Uma alternativa para controlar a oxidação do carbono em refratários seria seu recobrimento com óxidos $\left(\mathrm{Al}_{2} \mathrm{O}_{3}, \mathrm{SiO}_{2}\right.$, $\mathrm{TiO}_{2}, \mathrm{ZrO}_{2}$ ) ou carbeto de silício ( $\mathrm{SiC}$ ) sobre a superfície das partículas de carbono. Estes óxidos, tais como $\mathrm{TiO}_{2}$ e $\mathrm{ZrO}_{2}$, quando na presença do carbono, doam elétrons para o mesmo, gerando uma distribuição eletrônica estável que favorece a inibição da oxidação do carbono [15]. O carbono recoberto apresenta melhor resistência à oxidação, porém estudos indicam que em muitos casos este efeito é limitado, especialmente a elevadas temperaturas. Além disso, a fraca ligação entre o carbono e a camada de recobrimento, resulta na diminuição da resistência mecânica dos refratários [2].

Uma outra forma mais efetiva de prevenir a oxidação dos refratários contendo carbono seria o uso de substâncias conhecidas como antioxidantes, os quais reagem preferencialmente com o carbono diminuindo sua velocidade de consumo ou taxa de oxidação. Alguns dos produtos gerados pelas reações com os antioxidantes apresentam expansão volumétrica, o que reduz a porosidade do sistema e, assim, a permeabilidade e difusão do oxigênio no refratário.

\section{PRINCIPAIS ANTIOXIDANTES USADOS EM MATERIAIS REFRATÁRIOS}

Os antioxidantes mais utilizados em refratários são pós metálicos ( $\mathrm{Al}, \mathrm{Mg}, \mathrm{Si}$, ou ligas metálicas), carbetos ( $\mathrm{SiC}$, $\left.\mathrm{B}_{4} \mathrm{C}\right)$, boretos $\left(\mathrm{ZrB}_{2}, \mathrm{CaB}_{2}\right)$ ou uma combinação destes. A seleção do antioxidante varia de acordo com o material da matriz do refratário. Por exemplo, pós metálicos de $\mathrm{Mg}$ e $\mathrm{Al}$ são freqüentemente adicionados a refratários de $\mathrm{MgO}-\mathrm{C}$, enquanto $\mathrm{SiC}$ e $\mathrm{B}_{4} \mathrm{C}$ são usados em refratários de $\mathrm{Al}_{2} \mathrm{O}_{3}-\mathrm{C}$ [16]. Vários trabalhos foram desenvolvidos visando o entendimento do comportamento da atuação destes antioxidantes, porém a maioria destes estudos foram realizados para o sistema MgO-C [7-10, 17-22]. Cada antioxidante atua em diferentes faixas de temperatura assim, o uso de mais de um tipo de antioxidante pode complementar a ação destes materiais na prevenção da oxidação do carbono.

\section{Antioxidantes metálicos}

Os pós de $\mathrm{Al}, \mathrm{Mg}$, Si e suas ligas são muito usados como antioxidantes devido ao seu baixo custo e sua proteção efetiva, que proporciona um aumento na resistência mecânica e à oxidação dos refratários. Entretanto, sua utilização em concretos refratários contendo carbono é limitada pela sua forte tendência em reagir com água [17, 20, 22-24]. Assim, é necessário que a reação destes antioxidantes metálicos com a água durante o processamento dos concretos seja a mínima possível, a fim de que a maior parte do pó metálico possa atuar como inibidor da oxidação do carbono em elevadas temperaturas.

$\mathrm{Na}$ tentativa de viabilizar o uso de pós metálicos como antioxidantes em concretos refratários contendo carbono, diferentes tratamentos superficiais tem sido propostos na literatura como forma de inibir sua reação com a água. Um tratamento recomendado consiste na utilização de alcóxidos metálicos como precursores para a obtenção de um recobrimento a base de óxido $\left(\mathrm{Al}_{2} \mathrm{O}_{3}, \mathrm{SiO}_{2}\right.$ e $\left.\mathrm{TiO}_{2}\right)$ sobre o pó metálico [25-28]. Embora eficientes em evitar a oxidação dos pós metálicos, tais recobrimentos não resistem a etapa de mistura dos concretos, deixando novamente a superfície destes materiais exposta a ação da água e à geração do hidrogênio [28-30].

\section{Alumínio}

$\mathrm{Na}$ literatura é possível encontrar vários trabalhos dedicados ao estudo da atuação do alumínio como antioxidante [9, 10, 20-22, 31]. Nestes trabalhos existe um consenso de que há um valor ótimo na quantidade de $\mathrm{Al}$ a ser adicionado aos refratários e que deve-se considerar os seguintes aspectos: o tamanho das partículas do pó (recoberto ou não); as etapas de processamento do refratário; a porosidade inicial das peças.

$\mathrm{O}$ excesso de $\mathrm{Al}$ poderá levar a diminuição da resistência à corrosão e até a diminuição da resistência mecânica do material devido a formação de poros abertos. O teor ótimo varia na faixa de 3 a $5 \%$ de acordo com alguns fabricantes de refratários [6].

O comportamento dos diferentes antioxidantes como pó metálico de $\mathrm{Al}$ e suas ligas foram amplamente investigados em refratários de $\mathrm{MgO}-\mathrm{C}$, e a maioria destes estudos define a ordem das possíveis reações [20, 22, 32-34]:

O Al metálico oxida-se rapidamente depois do processo de preparação do refratário, inicialmente uma camada $\mathrm{Al}_{2} \mathrm{O}_{3}$ é formada no material (equação B).

$$
4 \mathrm{Al}_{(\mathrm{s})}+3 \mathrm{O}_{2(\mathrm{~g})} \leftrightarrow 2 \mathrm{Al}_{2} \mathrm{O}_{3(\mathrm{~s})}
$$

$\mathrm{Na}$ temperatura de fusão do $\mathrm{Al}\left(660^{\circ} \mathrm{C}\right)$, uma fina camada de $\mathrm{Al}_{2} \mathrm{O}_{3}$ pode aprisionar o $\mathrm{Al}$ líquido por um tempo, até esta se romper havendo a liberação do $\mathrm{Al}$ fundido. $\mathrm{O} \mathrm{Al}$ liberado reagirá com o carbono para formar $\mathrm{Al}_{4} \mathrm{C}_{3}$ (equação $\mathrm{C}$ ), que posteriormente poderá se combinar com o nitrogênio da atmosfera formando a fase AlN (equação D).

$$
\begin{aligned}
& 4 \mathrm{Al}_{(\mathrm{l}, \mathrm{g})}+3 \mathrm{C}_{(\mathrm{s})} \leftrightarrow \mathrm{Al}_{4} \mathrm{C}_{3(\mathrm{~s})} \\
& \mathrm{Al}_{4} \mathrm{C}_{3(\mathrm{~s})}+2 \mathrm{~N}_{2(\mathrm{~g})} \leftrightarrow 4 \mathrm{AlN}_{(\mathrm{s})}+3 \mathrm{C}_{(\mathrm{s})}
\end{aligned}
$$

$\mathrm{O} \mathrm{Al}_{4} \mathrm{C}_{3}$ e o $\mathrm{AlN}$ na temperatura de aproximadamente $1100{ }^{\circ} \mathrm{C}$ reagirão com o $\mathrm{CO}(\mathrm{g})$ (proveniente da oxidação direta do carbono e que se permeia pelo refratário) para formar $\mathrm{Al}_{2} \mathrm{O}_{3}$ (equações $\mathrm{E}-\mathrm{F}$ ). $\mathrm{O} \mathrm{Al}_{2} \mathrm{O}_{3}$ presente, em seguida reagirá com o $\mathrm{MgO}$ do refratário para formar o espinélio $\mathrm{MgAl}_{2} \mathrm{O}_{4}$ (equação $\mathrm{G}$ ).

$$
\mathrm{Al}_{4} \mathrm{C}_{3(\mathrm{~s})}+6 \mathrm{CO}_{(\mathrm{g})} \leftrightarrow 2 \mathrm{Al}_{2} \mathrm{O}_{3(\mathrm{~s})}+9 \mathrm{C}_{(\mathrm{s})}
$$




$$
\begin{aligned}
& 2 \mathrm{AlN}_{(\mathrm{s})}+3 \mathrm{CO}_{(\mathrm{g})} \leftrightarrow \mathrm{Al}_{2} \mathrm{O}_{3(\mathrm{~s})}+\mathrm{N}_{2(\mathrm{~g})}+3 \mathrm{C}_{(\mathrm{s})} \\
& \mathrm{Al}_{2} \mathrm{O}_{3(\mathrm{~s})}+\mathrm{MgO}_{(\mathrm{s})} \leftrightarrow \mathrm{MgAl}_{2} \mathrm{O}_{4(\mathrm{~s})}
\end{aligned}
$$

A formação de novos compostos devido a reação entre $\mathrm{Al}$ com o carbono, nitrogênio, oxigênio e o material refratário é acompanhada por um aumento no volume específico do sólido e resulta em um preenchimento parcial dos poros e redução da entrada dos gases para o interior do material, havendo assim a prevenção da oxidação do carbono.

A redução do $\mathrm{CO}(\mathrm{g})$ a $\mathrm{C}(\mathrm{s})$ contribui também para a inibição da oxidação do carbono e a formação de produtos de reação como o $\mathrm{Al}_{2} \mathrm{O}_{3}$, que melhoram a resistência mecânica dos materiais refratários. Experimentalmente foi observado que a formação de $\mathrm{Al}_{2} \mathrm{O}_{3}$ na superfície de $\mathrm{Al}_{4} \mathrm{C}_{3}$ e a formação de $\mathrm{MgAl}_{2} \mathrm{O}_{4}$ comprovam a oxidação do $\mathrm{Al}_{4} \mathrm{C}_{3}$ (Fig. 1a) Já a precipitação de "whiskers" de AIN na superfície dos grãos de $\mathrm{Al}_{4} \mathrm{C}_{3}$ indicam a transformação de $\mathrm{Al}_{4} \mathrm{C}_{3}$ a $\mathrm{AlN}$ (Fig. 1b).
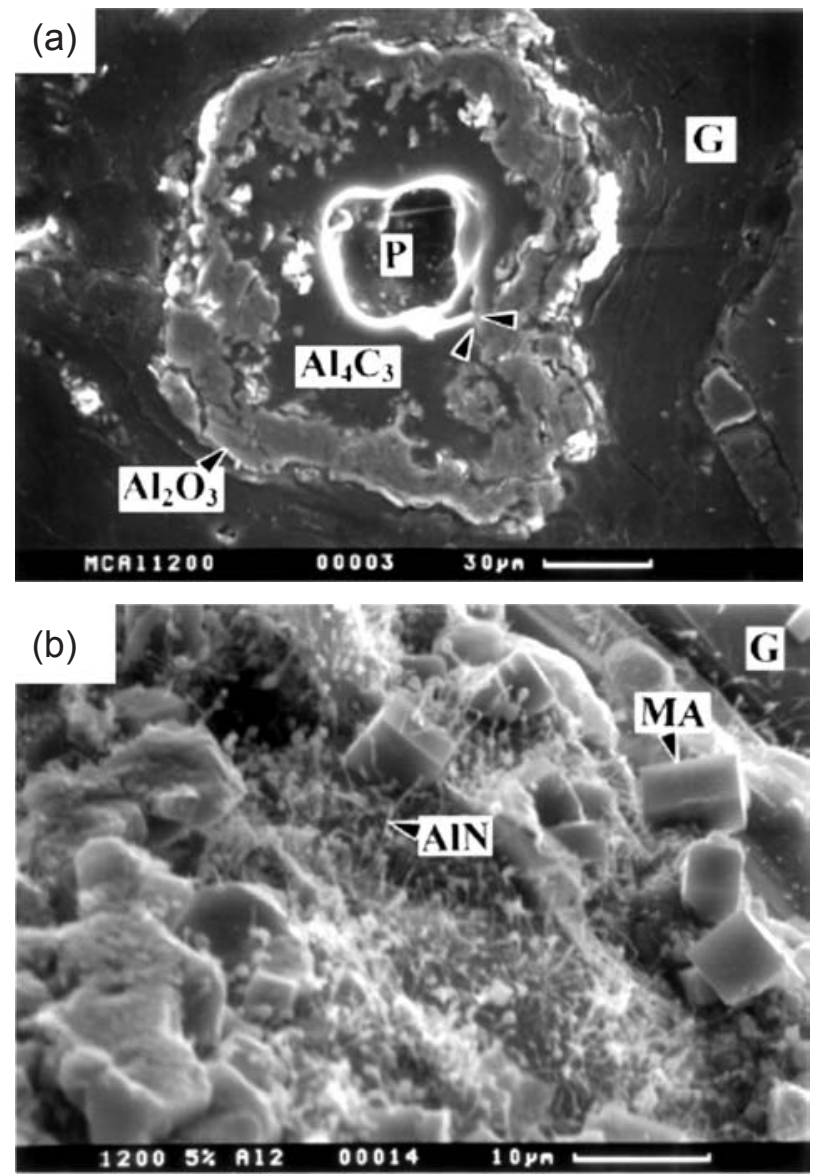

Figura 1: Microestrutura de tijolos de $\mathrm{MgO}-\mathrm{C}$ contendo $\mathrm{Al}$ queimados a $1200^{\circ} \mathrm{C}$ por $3 \mathrm{~h}$ em atmosfera redutora; (a) camada de $\mathrm{Al}_{2} \mathrm{O}_{3}$ formada sobre a fase $\mathrm{Al}_{4} \mathrm{C}_{3}$; (b) fases espinélio (MA) e AlN na superfície de fratura do tijolo. $\mathrm{G}=$ grafite, $\mathrm{P}=$ poro, $\mathrm{MA}=\mathrm{MgAl}_{2} \mathrm{O}_{4}$ [22]

[Figure 1: Microstructure of $\mathrm{MgO}-\mathrm{C}$ bricks containing $\mathrm{Al}$ heated at $1200{ }^{\circ} \mathrm{C}$ for $3 \mathrm{~h}$ in reducing atmosphere; (a) an $\mathrm{Al}_{4} \mathrm{C}_{3}$ core was coated by an $\mathrm{Al}_{2} \mathrm{O}_{3}$ layer; (b) (MA) spinel and $\mathrm{AlN}$ was found on the fracture surface of the brick. $G=$ graphite, $P=$ pore, $\left.M A=M_{g A l} O_{4}[22].\right]$
Entre 800 e $1000{ }^{\circ} \mathrm{C}$, a fase $\mathrm{Al}_{4} \mathrm{C}_{3}$ poderá reagir com o próprio $\mathrm{MgO}$ do refratário levando a formação da fase espinélio e de $\mathrm{Mg}(\mathrm{g})$ (equação $\mathrm{H}$ ). Já a $1300{ }^{\circ} \mathrm{C}$ também é possível a reação do $\mathrm{Al}$ metálico ainda presente na amostra com o $\mathrm{MgO}$, que também provoca a liberação de $\mathrm{Mg}(\mathrm{g})$ (equação I). Estas reações são prejudiciais às propriedades do refratário, pois a saída do $\mathrm{Mg}$ gasoso causa um aumento na porosidade aparente e assim diminuição da resistência mecânica e ao ataque por escória [21].

$$
\begin{gathered}
\mathrm{Al}_{4} \mathrm{C}_{3(\mathrm{~s})}+8 \mathrm{MgO}_{(\mathrm{s})} \leftrightarrow 2 \mathrm{Mg}_{2} \mathrm{Al}_{2} \mathrm{O}_{4(\mathrm{~s})}+3 \mathrm{C}_{(\mathrm{s})}+6 \mathrm{Mg}_{(\mathrm{g})} \\
2 \mathrm{H} \mathrm{Al}_{(\mathrm{l}, \mathrm{g})}+4 \mathrm{MgO}_{(\mathrm{s})} \leftrightarrow \mathrm{MgAl}_{2} \mathrm{O}_{4(\mathrm{~s})}+3 \mathrm{Mg}_{(\mathrm{g})}
\end{gathered}
$$

Para refratários que contem Si em sua composição, em temperaturas superiores a $1300{ }^{\circ} \mathrm{C}$ pode ocorrer também a reação entre $\mathrm{o} \mathrm{Al}_{2} \mathrm{O}_{3}$ e $\mathrm{o} \mathrm{SiO}_{2}$ havendo a formação de mulita $\left(\mathrm{Al}_{6} \mathrm{Si}_{2} \mathrm{O}_{13}\right)$. A formação de mulita é significante até $1500{ }^{\circ} \mathrm{C}$, porém com o aumento da temperatura ocorre a sua decomposição, e em $1600{ }^{\circ} \mathrm{C}$ praticamente há o desaparecimento desta fase [32]. Em temperaturas maiores que $1500^{\circ} \mathrm{C}$ observa-se não só a diminuição do teor de mulita e de carbono, mas também o aumento nos teores de $\mathrm{SiC}$ e $\mathrm{Al}_{2} \mathrm{O}_{3}$ nos refratários de $\mathrm{Al}_{2} \mathrm{O}_{3}-\mathrm{SiO}_{2}-\mathrm{SiC}-\mathrm{C}$ (equação $\mathrm{J}$ ).

$$
\mathrm{Al}_{6} \mathrm{Si}_{2} \mathrm{O}_{13(\mathrm{~s})}+6 \mathrm{C}_{(\mathrm{s})} \leftrightarrow 2 \mathrm{SiC}_{(\mathrm{s})}+3 \mathrm{Al}_{2} \mathrm{O}_{3(\mathrm{~s})}+4 \mathrm{CO}_{(\mathrm{g})}
$$

A decomposição da mulita provoca principalmente um aumento na porosidade aparente dos refratários e assim verifica-se uma diminuição da resistência mecânica e a corrosão destes materiais [32].

Para refratários de $\mathrm{MgO}-\mathrm{C}$, quando a temperatura é aumentada para $1500{ }^{\circ} \mathrm{C}$ a pressão parcial de $\mathrm{Al}(\mathrm{g})$ em equilíbrio com $\mathrm{Al}_{4} \mathrm{C}_{3}$ aumenta e o teor de $\mathrm{Mg}(\mathrm{g})$ torna-se significante devido a reação do $\mathrm{MgO}$ com $\mathrm{C}$ (equação $\mathrm{K}$ ). Assim, além da reação sólido-gás (equação L), a reação gásgás (equação M) também contribuirá para a formação de $\mathrm{MgAl}_{2} \mathrm{O}_{4}[17]$.

$$
\begin{gathered}
\mathrm{MgO}_{(\mathrm{s})}+\mathrm{C}_{(\mathrm{s})} \leftrightarrow \mathrm{Mg}_{(\mathrm{g})}+\mathrm{CO}_{(\mathrm{g})} \\
2 \mathrm{Al}_{(\mathrm{g})}+\mathrm{MgO}_{(\mathrm{s})}+3 \mathrm{CO}_{(\mathrm{g})} \leftrightarrow \mathrm{MgAl}_{2} \mathrm{O}_{4(\mathrm{~s})}+3 \mathrm{C}_{(\mathrm{s})} \\
2 \mathrm{Al}_{(\mathrm{g})}+\mathrm{Mg}_{(\mathrm{g})}+4 \mathrm{CO}_{(\mathrm{g})} \leftrightarrow \mathrm{MgAl}_{2} \mathrm{O}_{4(\mathrm{~s})}+4 \mathrm{C}_{(\mathrm{s})}
\end{gathered}
$$

A formação da fase espinélio é desejável não somente para atuar na prevenção da oxidação do carbono, mas também porque ela pode proporcionar um aumento da resistência mecânica dos materiais refratários.

Um dos problemas do uso de $\mathrm{Al}$ metálico como antioxidante persiste na formação da fase $\mathrm{Al}_{4} \mathrm{C}_{3}$, que é facilmente hidratada em temperatura ambiente (equação $\mathrm{N})$. A hidratação desta fase leva a formação de $\mathrm{Al}(\mathrm{OH})_{3}$ que provoca a expansão volumétrica e o possível aparecimento de trincas no material. A constatação de tal problema foi verificada em um tijolo usado de $\mathrm{MgO}-\mathrm{C}$, que foi coletado 
na planta de uma indústria siderúrgica e mantido exposto ao ar atmosférico por um longo período [35]. Pequenas trincas apareceram após 15 dias de exposição e com o aumento do tempo o tijolo gradualmente se desintegrou (Fig. 2). Este comportamento foi atribuído a hidratação da fase $\mathrm{Al}_{4} \mathrm{C}_{3}$ com conseqüente expansão volumétrica, provocando assim o aparecimento de defeitos.

$$
\mathrm{Al}_{4} \mathrm{C}_{3(\mathrm{~s})}+12 \mathrm{H}_{2} \mathrm{O}_{(\mathrm{g})} \leftrightarrow 3 \mathrm{CH}_{4(\mathrm{~g})}+4 \mathrm{Al}(\mathrm{OH})_{3(\mathrm{~s})}
$$

$\mathrm{O}$ teor de $\mathrm{Al}$ em refratários contendo carbono tem uma influência direta no comportamento de desintegração destes materiais quando expostos a umidade. Quanto maior a quantidade de $\mathrm{Al}$ adicionada ao refratário, maior será o aparecimento de trincas e mais rápido ocorrerá a desintegração da peça [20, 35].

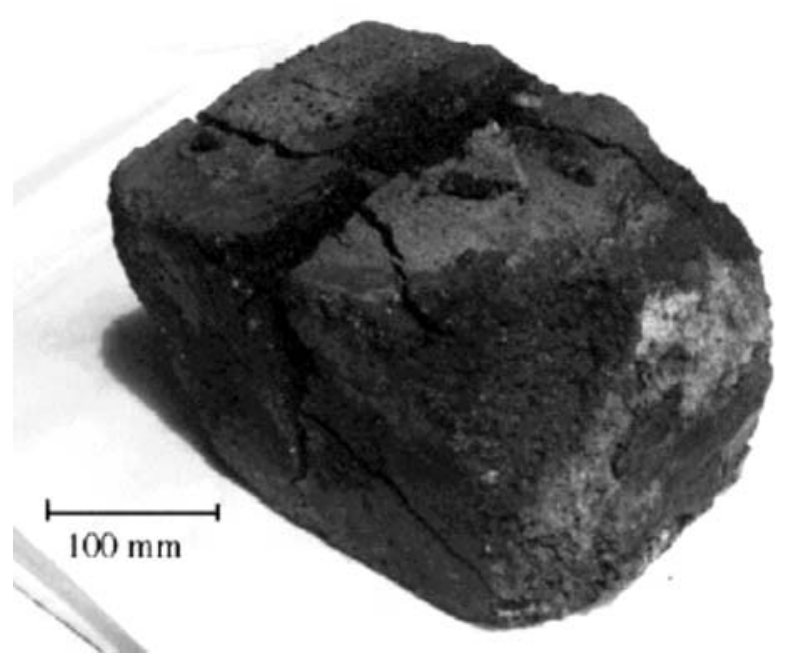

Figura 2: Fotografia de um típico tijolo de MgO-C desintegrado coletado de uma planta industrial depois de 20 dias de exposição ao ar atmosférico [35].

[Figure 2: Photograph of a typical disintegrated $\mathrm{MgO}-\mathrm{C}$ brick collected from one industrial plant after 20 days of exposure in open atmosphere [35].]

Alguns estudos sugerem que o pó de alumínio é mais eficaz do que os pós de magnésio e silício, entretanto cada antioxidante atua em diferentes faixas de temperatura e o uso de mais de um tipo de antioxidante pode complementar a ação destes materiais na prevenção da oxidação do carbono.

\section{Mg, Si e ligas metálicas}

Os estudos realizados para refratários com adições de $\mathrm{Mg}$ são em menor número do que para $\mathrm{Al}$. O perigo potencial do manuseio de tal pó pirofórico em um ambiente industrial, assim como sua disponibilidade tem levado ao uso mais freqüente de ligas de $\mathrm{Al}-\mathrm{Mg}$ [6]. Mesmo assim, $\mathrm{Mg}$ é considerado um bom antioxidante devido a sua facilidade em promover a formação de uma camada protetora densa na superfície dos refratários. A associação de $\mathrm{Al}-\mathrm{Mg}$ tem um efeito superior do que o uso do $\mathrm{Al}$ ou $\mathrm{Mg}$ isoladamente, pois além de prevenir a oxidação do carbono, a liga também promove uma maior resistência mecânica quando adicionado em conjunto com $\mathrm{Zr}$, SiC e $\mathrm{CaB}_{6}$ a refratários de $\mathrm{MgO}-\mathrm{C}$ e $\mathrm{Al}_{2} \mathrm{O}_{3}-\mathrm{C}[6,33,36]$.

Para os antioxidantes na forma de ligas metálicas, o mecanismo de reação pode ser considerado como uma combinação dos mecanismos de cada componente. Dependendo da reatividade de cada metal com $\mathrm{CO}(\mathrm{g})$ ou $\mathrm{O}_{2}(\mathrm{~g})$, eles atuarão em diferentes estágios. Por exemplo, se uma liga Al-Si é adicionada, o Al da liga age primeiramente como antioxidante, uma vez que o Al é esgotado o Si começa a atuar [22].

O pó de Si também tem sido usado com sucesso em materiais refratários [17, 20, 22, 32, 37]. Novamente, como o Al, há um teor ótimo de Si a ser adicionado a composição do refratário e as reações intermediárias são diferentes em natureza e ocorrem em temperaturas distintas [22].

Aproximadamente a $1200{ }^{\circ} \mathrm{C}$ o $\mathrm{Si}$ pode reagir com o C para formar $\mathrm{SiC}$ (equação $\mathrm{O}$ ).

$$
\mathrm{Si}_{(\mathrm{s})}+\mathrm{C}_{(\mathrm{s})} \leftrightarrow \quad \mathrm{SiC}_{(\mathrm{s})}
$$

O SiC formado se apresentará na forma de "whiskers" e ele atuará diretamente não só na prevenção da oxidação do carbono, mas também no aumento da resistência mecânica dos refratários [37].

Com o aumento da temperatura, o $\mathrm{SiC}$ reage com o nitrogênio ou $\mathrm{CO}(\mathrm{g})$ da atmosfera formando $\mathrm{Si}_{3} \mathrm{~N}_{4}$ e $\mathrm{SiO}_{2}$ respectivamente (equações $\mathrm{P}-\mathrm{Q}$ ). Posteriormente, $\mathrm{SiO}_{2}$ reagirá com o $\mathrm{MgO}$ do refratário para formar a fase $\mathrm{Mg}_{2} \mathrm{SiO}_{4}$ (equação R).

$$
\begin{aligned}
& 3 \mathrm{SiC}_{(\mathrm{s})}+2 \mathrm{~N}_{2(\mathrm{~g})} \leftrightarrow \mathrm{Si}_{3} \mathrm{~N}_{4(\mathrm{~s})}+3 \mathrm{C}_{(\mathrm{s})} \\
& \mathrm{SiC}_{(\mathrm{s})}+2 \mathrm{CO}_{(\mathrm{g})} \leftrightarrow \mathrm{SiO}_{2(\mathrm{~s})}+3 \mathrm{C}_{(\mathrm{s})} \\
& \mathrm{SiO}_{2(\mathrm{~s})}+2 \mathrm{MgO}_{(\mathrm{s})} \leftrightarrow \mathrm{Mg}_{2} \mathrm{SiO}_{4(\mathrm{~s})}
\end{aligned}
$$

A microestrutura de tijolos de $\mathrm{MgO}-\mathrm{C}$ contendo 5\%peso de Si (Fig. 3) e queimados em $1200{ }^{\circ} \mathrm{C}$ apresenta as fases $\mathrm{Si}, \mathrm{SiC}, \mathrm{Si}_{3} \mathrm{~N}_{4}, \mathrm{SiO}_{2}$ e $\mathrm{Mg}_{2} \mathrm{SiO}_{4}$, conforme previsto pelas equações P-R.

Depois que todo o $\mathrm{SiC}$ for transformado a $\mathrm{Si}_{3} \mathrm{~N}_{4}$ e/ou $\mathrm{SiO}_{2}, \mathrm{o} \mathrm{Si}_{3} \mathrm{~N}_{4}$ poderá ser oxidado a $\mathrm{SiO}_{2}$ (equação $\mathrm{S}$ ) o qual reagirá com $\mathrm{MgO}$ para formar $\mathrm{Mg}_{2} \mathrm{SiO}_{4}$, conforme descrito anteriormente na equação $\mathrm{R}$.

$$
\mathrm{Si}_{3} \mathrm{~N}_{4(\mathrm{~s})}+6 \mathrm{CO}_{(\mathrm{g})} \leftrightarrow 3 \mathrm{SiO}_{2(\mathrm{~s})}+6 \mathrm{C}_{(\mathrm{s})}+2 \mathrm{~N}_{2(\mathrm{~g})}
$$

Cálculos termodinâmicos revelam que o $\mathrm{Si}_{3} \mathrm{~N}_{4}$ torna-se instável a partir de $1500{ }^{\circ} \mathrm{C}$ e com o aumento da temperatura ele irá ser convertido novamente em $\mathrm{SiC}$ [17]. Na temperatura de $1500^{\circ} \mathrm{C}$ a pressão parcial de $\mathrm{SiO}(\mathrm{g})$ (equação T) torna-se significante e como no processo de formação do $\mathrm{MgAl}_{2} \mathrm{O}_{4}$, 

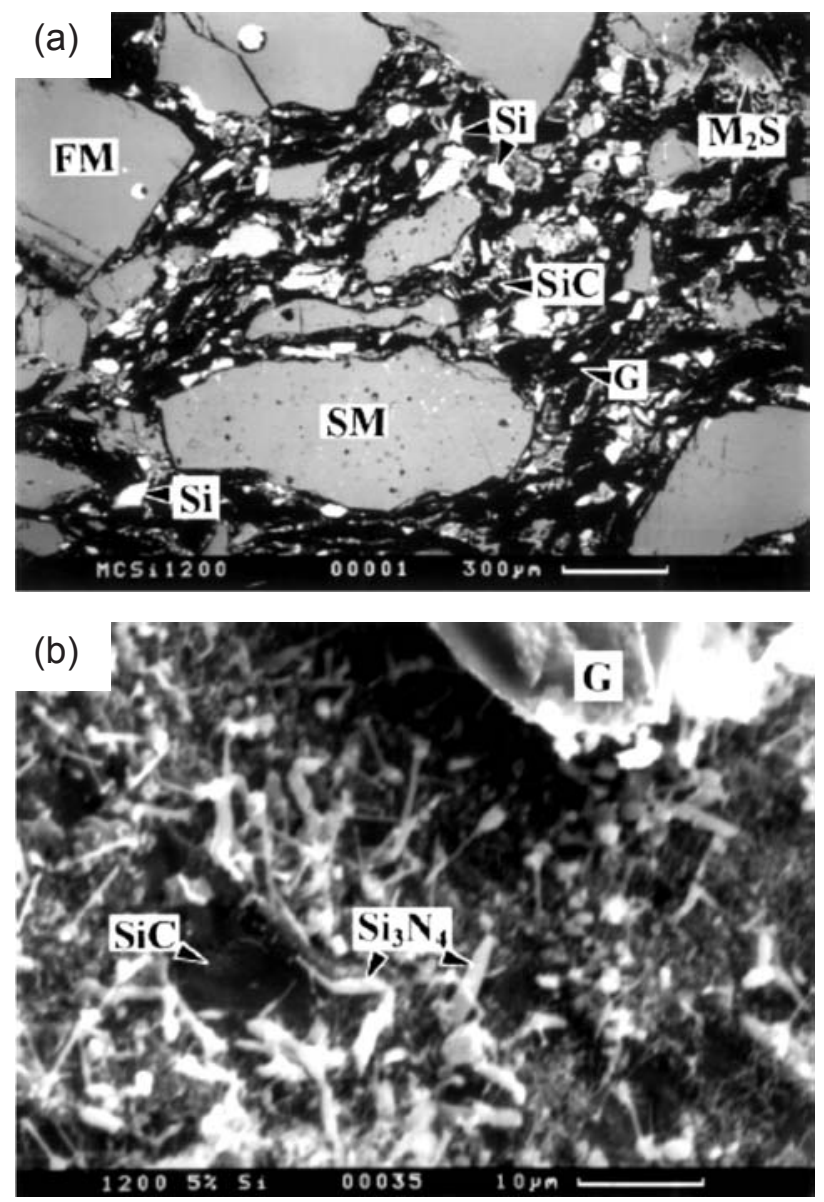

Figura 3: Microestrutura de tijolos de $\mathrm{MgO}-\mathrm{C}$ contendo $\mathrm{Si}$ queimados a $1200{ }^{\circ} \mathrm{C}$ por $3 \mathrm{~h}$ em atmosfera redutora; (a) muitos grãos de $\mathrm{Si}$ ainda permanecem na amostra, embora $\mathrm{SiC}, \mathrm{SiO}_{2}$ e $\mathrm{Mg}_{2} \mathrm{SiO}_{4}$ começam a aparecer; (b) Whiskers de $\mathrm{Si}_{3} \mathrm{~N}_{4}$ são observados na superfície de fratura do tijolo. $\mathrm{G}=$ grafite, $\mathrm{FM}=\mathrm{MgO}$ fundida, $\mathrm{SM}=\mathrm{MgO}$ sinterizada, $\mathrm{M}_{2} \mathrm{~S}=\mathrm{Mg}_{2} \mathrm{SiO}_{4}[22]$

[Figure 3: Microstructure of $\mathrm{MgO}-\mathrm{C}$ bricks containing Si heated at $1200{ }^{\circ} \mathrm{C}$ for $3 \mathrm{~h}$ in reducing atmosphere; (a) many unreacted $\mathrm{Si}$ grains still remained in the sample, although $\mathrm{SiC}, \mathrm{SiO}_{2}$ and $\mathrm{Mg}_{2} \mathrm{SiO}_{4}$ were detected; (b) $\mathrm{Si}_{3} \mathrm{~N}_{4}$ whiskers observed on the fracture surface of the brick $G=$ graphite, $F M=$ fused $M g O, S M=$ sintered $\left.\mathrm{MgO}, \mathrm{M}_{2} \mathrm{~S}=\mathrm{Mg}_{2} \mathrm{SiO}_{4}[22].\right]$

o $\mathrm{Mg}_{2} \mathrm{SiO}_{4}$ pode ser formado via reação sólido-gás (equação U) ou gás-gás (equação V).

$$
\begin{aligned}
& \mathrm{SiC}_{(\mathrm{s})}+\mathrm{CO}_{(\mathrm{g})} \leftrightarrow \mathrm{SiO}_{(\mathrm{g})}+2 \mathrm{C}_{(\mathrm{s})} \\
& \mathrm{SiO}_{(\mathrm{g})}+2 \mathrm{MgO}_{(\mathrm{s})}+\mathrm{CO}_{(\mathrm{g})} \leftrightarrow \mathrm{Mg}_{2} \mathrm{SiO}_{4(\mathrm{~s})}+\mathrm{C}_{(\mathrm{s})} \\
& \mathrm{SiO}_{(\mathrm{g})}+2 \mathrm{Mg}_{(\mathrm{g})}+3 \mathrm{CO}_{(\mathrm{g})} \leftrightarrow \mathrm{Mg}_{2} \mathrm{SiO}_{4(\mathrm{~s})}+3 \mathrm{C}_{(\mathrm{s})}
\end{aligned}
$$

Para explicar o comportamento de resistência à oxidação das amostras contendo silício também deve ser mencionado que, dependendo da pressão parcial de oxigênio e temperatura de oxidação o processo pode ser classificado como ativo ou passivo [38].
A oxidação ativa ocorre com a remoção de $\mathrm{SiO}_{(\mathrm{g})}$ da amostra. Durante o processo de oxidação há um aumento na pressão de $\mathrm{CO}(\mathrm{g})$ dentro da amostra devido a reação do carbono com o oxigênio. Assim, a oxidação de SiC dentro da amostra torna-se ativa através da reação descrita pela equação (T). Por outro lado, na oxidação passiva uma fina camada de $\mathrm{SiO}_{2}$ forma-se nas partículas de $\mathrm{SiC}$ provocando uma diminuição da oxidação. Devido a alta pressão parcial de $\mathrm{O}_{2}$ na atmosfera do forno, a pressão nas regiões próximas da superfície da amostra também será alta e assim a seguinte reação poderá ocorrer (equação $\mathrm{W}$ ):

$$
\mathrm{SiO}_{(\mathrm{g})}+0,5 \mathrm{O}_{2(\mathrm{~g})} \leftrightarrow \quad \mathrm{SiO}_{2(\mathrm{~s})}
$$

A formação de $\mathrm{SiO}_{2}$ próximo a superfície terá um efeito retardador na oxidação do refratário. E quanto maior o teor de $\mathrm{Si}$ adicionado a composição menor será a espessura desta camada de $\mathrm{SiO}_{2}$ e assim a taxa de oxidação será controlada pela difusão do oxigênio através desta [38].

A adição de Si como antioxidante tem um efeito positivo na resistência a oxidação e a corrosão do material refratário devido a grande formação da fase $\mathrm{Mg}_{2} \mathrm{SiO}_{4}$ [20]. Esta fase atua da mesma forma que o $\mathrm{MgAl}_{2} \mathrm{O}_{4}$ que graças a sua expansão volumétrica provoca a formação de uma camada com menor porosidade na superfície do material, evitando a difusão do oxigênio e da escória para seu interior.

\section{Antioxidantes não-metálicos}

O uso de antioxidantes não metálicos é freqüentemente associado com a formação de fases líquidas, provenientes da reação destes materiais com o oxigênio e dos óxidos formados com os outros componentes do refratário a elevadas temperaturas. Tais líquidos atuam no preenchimento dos poros abertos e consequentemente na prevenção da oxidação do carbono. Entretanto, a formação destas fases afeta negativamente a resistência à corrosão e a resistência mecânica dos refratários a elevadas temperaturas.

$$
\text { Carbetos ( } \left.\mathrm{SiC}, \mathrm{B}_{4} \mathrm{C}, \mathrm{Al}_{8} \mathrm{~B}_{4} \mathrm{C}_{7}, \mathrm{Al}_{4} \mathrm{SiC}_{4}\right)
$$

O SiC possui a tendência em formar a fase $\mathrm{SiO}_{2}$ sob condições oxidantes e sua ação como agente bloqueador dos poros dos tijolos refratários é bem estudada, pelo menos acima de $1500^{\circ} \mathrm{C}[6,16]$. A atuação do $\mathrm{SiC}$ como antioxidante em materiais refratários, é similar aos mecanismos descritos anteriormente para o Si, conforme as equações (P-W). Assim como para outros antioxidantes, existe um teor ótimo de $\mathrm{SiC}$ a ser adicionado em uma composição de um refratário. Quando a quantidade de $\mathrm{SiC}$ é maior do que 5\%-peso, a vida útil de refratários de $\mathrm{MgO}-\mathrm{C}$ diminui devido a formação excessiva de $\mathrm{SiO}_{2}(\mathrm{~s})$ e assim a resistência a corrosão é prejudicada [17, 22]. Alguns estudos demonstram que o uso de $\mathrm{Si}$ é mais efetivo do que o $\mathrm{SiC}$ em refratário de $\mathrm{MgO}$ $\mathrm{C}$, pois o primeiro permite a formação da fase $\mathrm{Mg}_{2} \mathrm{SiO}_{4} \mathrm{em}$ maior quantidade e assim, melhores serão as propriedades do material [20]. 
Antioxidantes, como o $\mathrm{B}_{4} \mathrm{C}$, não agem somente como agentes oxidantes de $\mathrm{CO}(\mathrm{g})$, mas também estimulam a formação de uma camada óxida densa na superfície quente do refratário que atua na prevenção da oxidação do carbono. Cálculos termodinâmicos indicam que o $\mathrm{B}_{4} \mathrm{C}$ pode reagir com o oxigênio da atmosfera até mesmo antes do carbono em temperaturas menores que $600{ }^{\circ} \mathrm{C}$ (equação X) [38]. Análises termogravimétricas comprovam que a oxidação ocorre na seguinte seqüência: $\mathrm{B}_{4} \mathrm{C}$, carbono, $\mathrm{Al}-\mathrm{Si}, \mathrm{Al}, \mathrm{SiC}$ [19]. A aproximadamente $1000{ }^{\circ} \mathrm{C}$ o $\mathrm{B}_{4} \mathrm{C}$ pode reagir com o $\mathrm{CO}(\mathrm{g})$ ou $\mathrm{N}_{2}(\mathrm{~g})$ da atmosfera para formar $\mathrm{B}_{2} \mathrm{O}_{3}$ ou $\mathrm{BN}$, respectivamente (equações Y-Z).

$$
\begin{aligned}
& \mathrm{B}_{4} \mathrm{C}_{(\mathrm{s})}+3 \mathrm{O}_{2(\mathrm{~g})} \leftrightarrow 2 \mathrm{~B}_{2} \mathrm{O}_{3(\mathrm{l})}+\mathrm{C}_{(\mathrm{s})} \\
& \mathrm{B}_{4} \mathrm{C}_{(\mathrm{s})}+6 \mathrm{CO}_{(\mathrm{g})} \leftrightarrow 2 \mathrm{~B}_{2} \mathrm{O}_{3(\mathrm{l})}+7 \mathrm{C}_{(\mathrm{s})} \\
& \mathrm{B}_{4} \mathrm{C}_{(\mathrm{s})}+2 \mathrm{~N}_{2(\mathrm{~g})} \leftrightarrow 4 \mathrm{BN}_{(\mathrm{s})}+\mathrm{C}_{(\mathrm{s})}
\end{aligned}
$$

$\mathrm{O} \quad \mathrm{B}_{2} \mathrm{O}_{3}$ formado ainda poderá reagir com o $\mathrm{MgO}$ para formar a fase $3 \mathrm{MgO} \cdot \mathrm{B}_{2} \mathrm{O}_{3}$ (equação AA), cujo ponto de fusão é $1360{ }^{\circ} \mathrm{C}$. A formação da fase $3 \mathrm{MgO} \cdot \mathrm{B}_{2} \mathrm{O}_{3}$ é rápida e, em apenas $15 \mathrm{~min}$, na temperatura de $1000^{\circ} \mathrm{C}$ a reação é praticamente completada $[20,22,33]$.

$$
\mathrm{B}_{2} \mathrm{O}_{3(1)}+3 \mathrm{MgO}_{(\mathrm{s})} \leftrightarrow \quad 3 \mathrm{MgO} \cdot \mathrm{B}_{2} \mathrm{O}_{3(1)}
$$

$\mathrm{B}_{4} \mathrm{C}$ é um antioxidante efetivo e susceptível a oxidação, porém assim como os demais antioxidantes a base de boro, ele é caro e a formação das fases líquidas $\mathrm{B}_{2} \mathrm{O}_{3}$ e $3 \mathrm{MgO} \cdot \mathrm{B}_{2} \mathrm{O}_{3}$ a altas temperaturas também diminui a resistência mecânica e à corrosão do refratário [38-40].

A avaliação da atuação dos antioxidantes pode ser feita verificando-se a perda de carbono sofrida pelos materiais

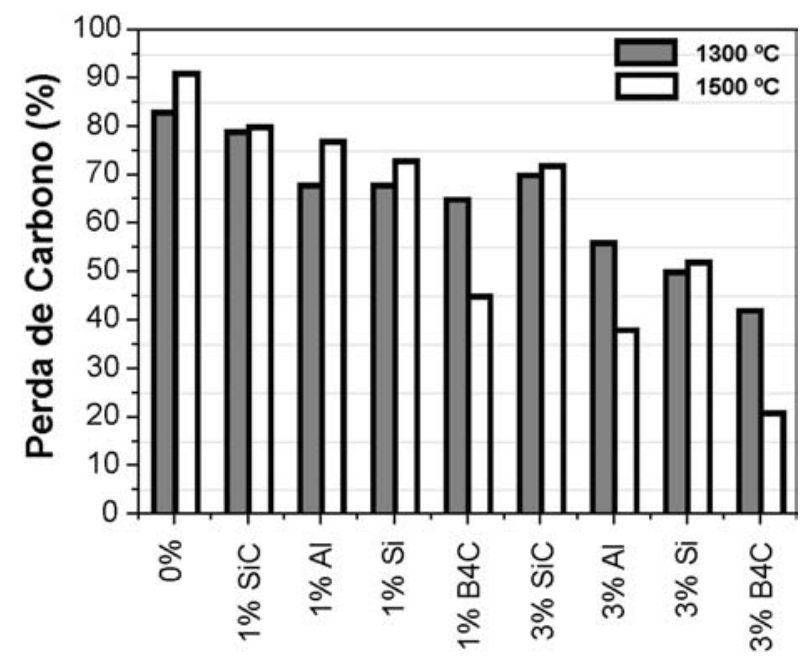

Figura 4: Porcentagem de perda de carbono de amostras de refratários de $\mathrm{MgO}-\mathrm{C}$ com e sem antioxidantes, oxidadas a 1300 e $1500{ }^{\circ} \mathrm{C}$ por $6 \mathrm{~h}[20]$.

[Figure 4: Carbon loss in $\mathrm{MgO}-\mathrm{C}$ refractory specimens with and without antioxidants, oxidized for $6 \mathrm{~h}$ at 1300 and $1500{ }^{\circ} \mathrm{C}$ [20].]

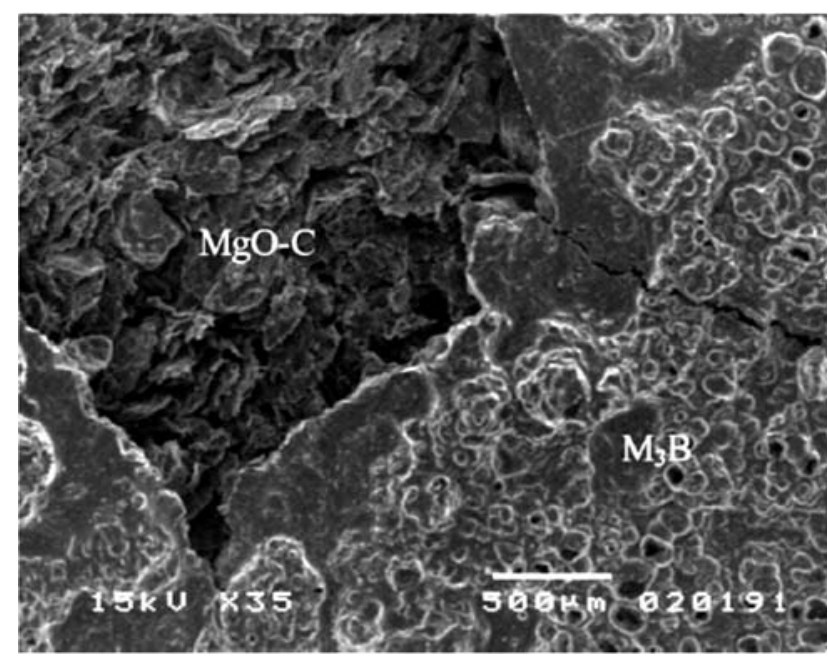

Figura 5: Micrografia da superfície de um tijolo refratário de $\mathrm{MgO}-$ $\mathrm{C}$ contendo $3 \%$-peso de $\mathrm{B}_{4} \mathrm{C}$ após teste de oxidação a $1500{ }^{\circ} \mathrm{C}$ por 6 h. $\mathrm{M}_{3} \mathrm{~B}=3 \mathrm{MgO} \cdot \mathrm{B}_{2} \mathrm{O}_{3}[20]$.

[Figure 5: Micrograph of the surface of the $3 \mathrm{wt} \% \mathrm{~B} 4 \mathrm{C}$ added $\mathrm{MgO}-\mathrm{C}$ refractory specimen oxidized for $6 \mathrm{~h}$ at $\quad 1500^{\circ} \mathrm{C} . M_{3} B=$ $3 \mathrm{MgO} . \mathrm{B}_{2} \mathrm{O}_{3}[20]$.]

refratários. Uma comparação entre vários antioxidantes é mostrada na Fig. 4. Pode-se observar que o $\mathrm{B}_{4} \mathrm{C}$ é o antioxidante mais efetivo, seguido pelo $\mathrm{Si}, \mathrm{Al}$ e $\mathrm{SiC}$. O $\mathrm{B}_{4} \mathrm{C}$ apresentou inclusive um comportamento melhor na temperatura de $1500{ }^{\circ} \mathrm{C}$, quando comparado com os resultados obtidos a $1300{ }^{\circ} \mathrm{C}$. Acredita-se que isto ocorre devido a formação da fase $3 \mathrm{MgO} \cdot \mathrm{B}_{2} \mathrm{O}_{3}$ que acima do seu ponto de fusão atua como uma barreira ao oxigênio [20]. É definido que esta fase no seu estado líquido preenche os poros e forma uma fina camada sobre a superfície do refratário em $1500{ }^{\circ} \mathrm{C}$ (Fig. 5), assim o oxigênio presente não pode se difundir para o interior do material.

Resultados recentes indicam que a combinação de pó de alumínio recoberto e $\mathrm{B}_{4} \mathrm{C}$ é uma das alternativas mais efetivas para a diminuição da oxidação do carbono em concretos refratários [6, 19, 21].

Embora seja claro que o Al metálico pode efetivamente inibir a oxidação de refratários contendo carbono, a sua tendência à formação da fase $\mathrm{Al}_{4} \mathrm{C}_{3}$ consiste em um fator prejudicial, pois pode levar a hidratação e o aparecimento de defeitos mesmo a temperatura ambiente. Desta forma, uma nova idéia consiste na combinação de $\mathrm{Al}$ com carbetos para produzir compostos complexos, tais como $\mathrm{Al}_{8} \mathrm{~B}_{4} \mathrm{C}_{7} \mathrm{e}$ $\mathrm{Al}_{4} \mathrm{SiC}_{4}$ que apresentam excelente resistência à hidratação e inibem a oxidação do carbono [41-44].

As possíveis transformações do $\mathrm{Al}_{8} \mathrm{~B}_{4} \mathrm{C}_{7}$ iniciam-se entre $1000-1200{ }^{\circ} \mathrm{C}$, mas tornam-se rápidas a partir de $1400{ }^{\circ} \mathrm{C}$. Inicialmente $\mathrm{o}_{8} \mathrm{~B}_{4} \mathrm{C}_{7}$ reage com o $\mathrm{CO}(\mathrm{g})$ formando as fases $\mathrm{Al}_{2} \mathrm{O}_{3}, \mathrm{~B}_{2} \mathrm{O}_{3}$ e $\mathrm{C}$ (equação $\mathrm{AB}$ ). Com o aumento da temperatura, a fase $\mathrm{B}_{2} \mathrm{O}_{3}$ poderá reagir com o $\mathrm{MgO}$ ou $\mathrm{Al}_{2} \mathrm{O}_{3}$ dos refratários para formar $3 \mathrm{MgO} \cdot \mathrm{B}_{2} \mathrm{O}_{3}$ (equação $\mathrm{AA}$ ) ou $\mathrm{Al}_{18} \mathrm{~B}_{4} \mathrm{O}_{33}$ (equação $\mathrm{AC}$ ), respectivamente.

$$
\mathrm{Al}_{8} \mathrm{~B}_{4} \mathrm{C}_{7(\mathrm{~s})}+18 \mathrm{CO}_{(\mathrm{s})} \leftrightarrow 4 \mathrm{Al}_{2} \mathrm{O}_{3(\mathrm{~s})}+2 \mathrm{~B}_{2} \mathrm{O}_{3(1)}+25 \mathrm{C}_{(\mathrm{s})}
$$




$$
36 \mathrm{Al}_{2} \mathrm{O}_{3(\mathrm{~s})}+4 \mathrm{~B}_{2} \mathrm{O}_{3(\mathrm{l})} \leftrightarrow 2 \mathrm{Al}_{18} \mathrm{~B}_{4} \mathrm{O}_{33(\mathrm{~s})}
$$

De acordo com os estudos realizados até o momento o $\mathrm{Al}_{8} \mathrm{~B}_{4} \mathrm{C}_{7}$ é até mesmo mais efetivo do que a combinação Al$\mathrm{B}_{4} \mathrm{C}$, na proteção a oxidação do carbono. Experimentalmente foi verificado que amostras de refratários de $\mathrm{MgO}-\mathrm{C}$ contendo $\mathrm{Al}_{8} \mathrm{~B}_{4} \mathrm{C}_{7}$, após testes de oxidação em diferentes temperaturas apresentaram uma estreita camada sem carbono na superfície das peças, confirmando assim a sua excelente atuação como antioxidante (Fig. 6).

Já as transformações de fases do $\mathrm{Al}_{4} \mathrm{SiC}_{4}$ ocorrem a partir da reação com o $\mathrm{CO}(\mathrm{g})$ para formar $\mathrm{Al}_{2} \mathrm{O}_{3}, \mathrm{SiC}$ e $\mathrm{C}$ (equação $\mathrm{AD}$ ). Quando o $\mathrm{Al}_{4} \mathrm{SiC}_{4}$ reage totalmente, o $\mathrm{SiC}$ e a $\mathrm{Al}_{2} \mathrm{O}_{3}$ formados reagirão com o $\mathrm{CO}(\mathrm{g})$ para formar

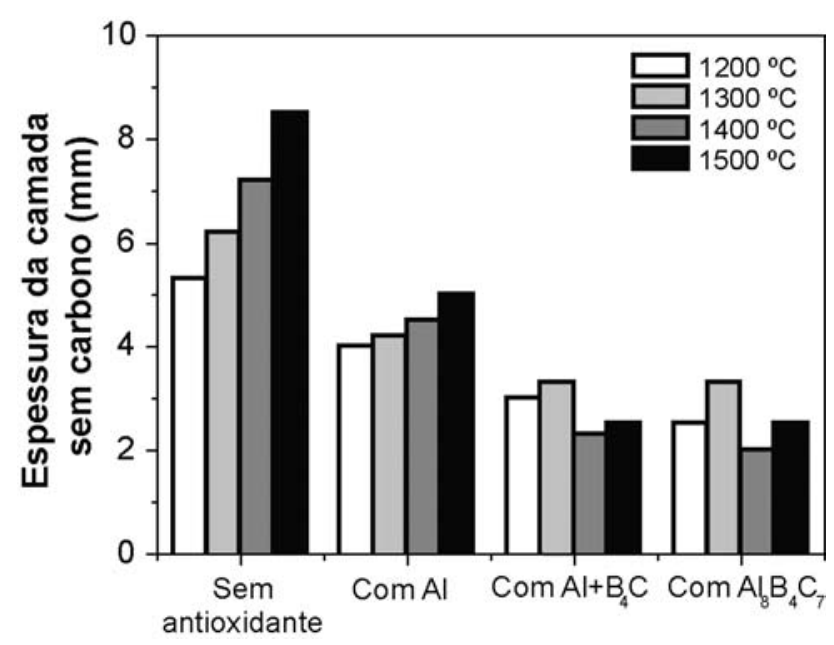

Figura 6: Espessura da camada sem carbono das amostras de MgO-C queimadas em diferentes temperaturas por $3 \mathrm{~h}$ ao ar [44]. [Figure 6: Thickness of the decarbonized layers of the $\mathrm{MgO}-\mathrm{C}$ samples heated at different temperatures for $3 h$ in air [44].] mulita $\left(\mathrm{Al}_{6} \mathrm{Si}_{2} \mathrm{O}_{13}\right)$ (equação $\mathrm{AE}$ ) e carbono, em temperaturas menores que $1560{ }^{\circ} \mathrm{C}$ [45].

$$
\begin{gathered}
\mathrm{Al}_{4} \mathrm{SiC}_{4(\mathrm{~s})}+6 \mathrm{CO}_{(\mathrm{g})} \leftrightarrow 2 \mathrm{Al}_{2} \mathrm{O}_{3(\mathrm{~s})}+\mathrm{SiC}_{(\mathrm{s})}+9 \mathrm{C}_{(\mathrm{s})} \\
3 \mathrm{Al}_{2} \mathrm{O}_{3(\mathrm{~s})}+2 \mathrm{SiC}_{(\mathrm{s})}+4 \mathrm{CO}_{(\mathrm{g})} \leftrightarrow \mathrm{Al}_{6} \mathrm{Si}_{2} \mathrm{O}_{13(\mathrm{~s})}+6 \mathrm{C}_{(\mathrm{s})}
\end{gathered}
$$

As mudanças das fases de um pó de $\mathrm{Al}_{4} \mathrm{SiC}_{4}$ quando aquecido em atmosfera redutora são mostradas na Fig. 7, comprovando o aparecimento das fases previstas nas equações $\mathrm{AD}$ e AE.

Testes experimentais realizados para composições de refratários de $\mathrm{MgO}-\mathrm{C}$ e $\mathrm{Al}_{2} \mathrm{O}_{3}-\mathrm{C}$ contendo 5\%-peso de $\mathrm{Al}_{4} \mathrm{SiC}_{4}$ mostraram excelentes resultados na prevenção a oxidação do carbono, com aumento na resistência mecânica dos refratários (Fig. 8) devido principalmente a formação da fase espinélio e mulita e nenhuma tendência a hidratação $[2,45,46]$. Os compostos $\mathrm{Al}_{8} \mathrm{~B}_{4} \mathrm{C}_{7}$ e $\mathrm{Al}_{4} \mathrm{SiC}_{4}$ são dois possíveis candidatos a serem utilizados como antioxidantes, porém a sua produção em grande quantidade pode ser economicamente inviável [2].

\section{Boretos $(\mathrm{ZrB}, \mathrm{Mg}-\mathrm{B}, \mathrm{CaB})$}

Os antioxidantes a base de boro atuam para bloquear os poros dos refratários e reduzir a oxidação do carbono pela formação de fases líquidas, como descrito anteriormente para o $\mathrm{B}_{4} \mathrm{C}$ [22]. Durante os últimos anos muitos testes bem sucedidos foram relatados usando diferentes fontes de boro [17, 20, 22, 31, 32, 46]. Porém, estes antioxidantes são caros e a formação de fases líquidas a altas temperaturas também diminui a resistência mecânica e à corrosão dos refratários quando submetidos a altas temperaturas.

$\mathrm{O}$ efeito da adição de $\mathrm{ZrB}_{2}$ em tijolos refratários de $\mathrm{MgO}-\mathrm{C}$ e $\mathrm{Al}_{2} \mathrm{O}_{3}-\mathrm{C}$ foi estudado principalmente nas temperaturas entre 600 e $1200^{\circ} \mathrm{C}[46]$. De acordo com dados

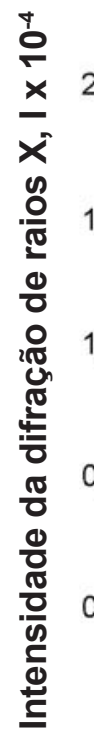

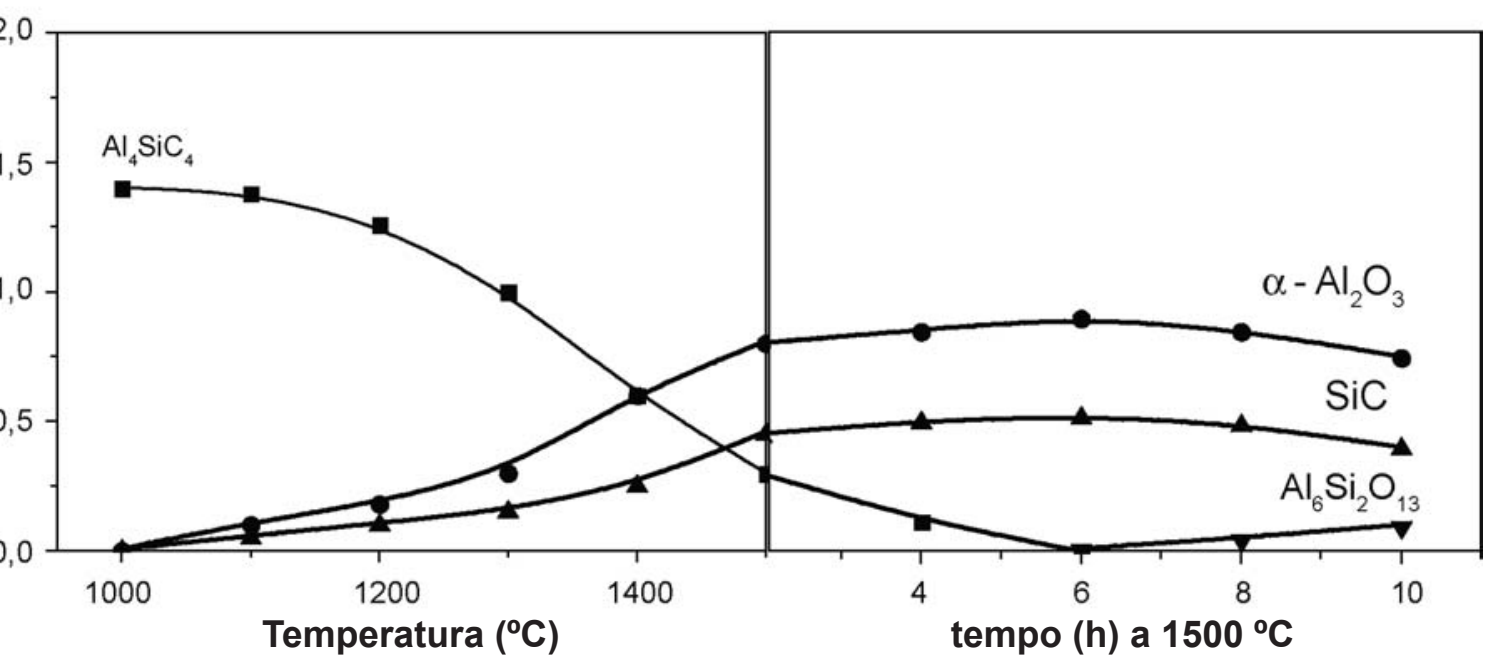

Figura 7: Mudanças de fases do pó de $\mathrm{Al}_{4} \mathrm{SiC}_{4}$ aquecido em atmosfera redutora em várias temperaturas e a $1500{ }^{\circ} \mathrm{C}$ de 2 a $10 \mathrm{~h}[45]$. [Figure 7: Phase changes of the $\mathrm{Al}_{4} \mathrm{SiC}_{4}$ powder heated in reducing atmosphere at various temperatures and at $1500{ }^{\circ} \mathrm{C}$ for 2 to $10 \mathrm{~h}$ [45].] 


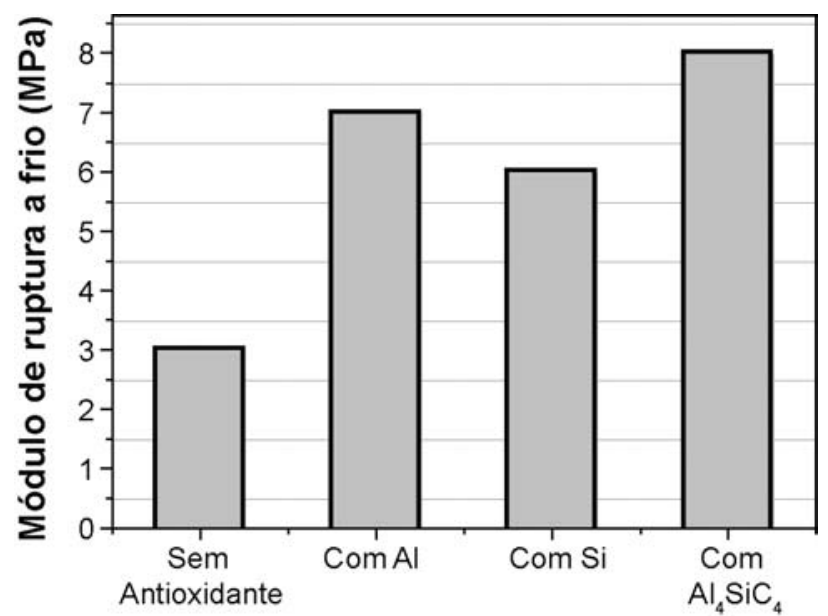

Figura 8: Módulo de ruptura à frio de tijolos de $\mathrm{Al}_{2} \mathrm{O}_{3}-\mathrm{C}$ contendo vários antioxidantes [2].

[Figure 8: Cold modulus of rupture of the $\mathrm{Al}_{2} \mathrm{O}_{3}$-C bricks containing various antioxidants [2].]

termodinâmicos a partir de $650{ }^{\circ} \mathrm{C}$ o $\mathrm{ZrB}_{2}$ atua na redução do $\mathrm{CO}(\mathrm{g})$, proveniente da oxidação direta do carbono, para formar $\mathrm{ZrO}_{2}, \mathrm{~B}_{2} \mathrm{O}_{3}$ e C (equação $\mathrm{AF}$ ).

$$
\mathrm{ZrB}_{2(\mathrm{~s})}+5 \mathrm{CO}_{(\mathrm{g})} \leftrightarrow \quad \mathrm{ZrO}_{2(\mathrm{~s})}+\mathrm{B}_{2} \mathrm{O}_{3(1)}+5 \mathrm{C}_{(\mathrm{s})}
$$

Os óxidos obtidos a partir da reação do $\mathrm{ZrB}_{2}$ com $\mathrm{CO}(\mathrm{g})$ tendem a formar uma camada protetora na superfície do material contribuindo para a redução da oxidação do carbono. Entretanto em temperaturas maiores que 1200 ${ }^{\circ} \mathrm{C}$ a fase $\mathrm{B}_{2} \mathrm{O}_{3}$ torna-se instável e a inibição a oxidação do carbono diminui [46]. Ainda são necessários estudos mais criteriosos para se determinar qual a influência do uso de $\mathrm{ZrB}_{2}$ em outras propriedades dos materiais refratários além do seu papel na prevenção da oxidação do carbono.

A adição simultânea de dois antioxidantes como $\mathrm{Al}^{-} \mathrm{B}_{4} \mathrm{C}$, $\mathrm{Mg}-\mathrm{B}$ e Al-CaB têm sido estudada [22, 36, 47-49].

$\mathrm{O}$ estudo da adição de $\mathrm{Mg}$-B juntamente com $\mathrm{Al}$ ou ligas $\mathrm{Al}-\mathrm{Mg}$ para atuarem como antioxidantes comprovou a boa atuação destes materiais na inibição da oxidação do carbono e no aumento do módulo de ruptura a quente dos refratários de $\mathrm{MgO}-\mathrm{C}$ e $\mathrm{Al}_{2} \mathrm{O}_{3}-\mathrm{C}[48,49]$. De acordo com resultados experimentais [49], o $\mathrm{Mg}-\mathrm{B}$ pode reagir a partir da temperatura de $1000{ }^{\circ} \mathrm{C}$ com o $\mathrm{CO}(\mathrm{g})$ presente na atmosfera levando a formação de $\mathrm{B}_{2} \mathrm{O}_{3}(1)$ e $\mathrm{B}_{4} \mathrm{C}(\mathrm{s})$. $\mathrm{O}$ uso combinado de $\mathrm{Mg}-\mathrm{B}$ com $\mathrm{Al}$ ou ligas $\mathrm{Al}-\mathrm{Mg}$ proporciona a formação de uma camada óxida na superfície do refratário consistindo principalmente do espinélio $\mathrm{MgAl}_{2} \mathrm{O}_{4}, \mathrm{MgO}$ e de uma fase vítrea a base de boro. $\mathrm{O} \mathrm{Mg}-\mathrm{B}$ atua como um eficiente antioxidante em temperaturas menores que $1000{ }^{\circ} \mathrm{C}$, porém acima deste valor sua atuação é pouco evidenciada [49].

$\mathrm{A}$ adição de $\mathrm{CaB}_{6}$ a tijolos refratários de $\mathrm{MgO}-\mathrm{C}$ contendo $\mathrm{Al}$ e ligas de $\mathrm{Al}-\mathrm{Mg}$ também contribui para reduzir a oxidação do carbono e para a melhoria das propriedades do material. Observou-se que o $\mathrm{CaB}_{6}$ pode reagir com o carbono ou $\mathrm{CO}(\mathrm{g})$ a partir de $1000{ }^{\circ} \mathrm{C}$ para formar $\mathrm{CaB}_{2} \mathrm{C}_{2} \mathrm{e}$ $\mathrm{CaC}_{2}$, como descrito pelas equações AG-AH.

$$
\begin{aligned}
& \mathrm{CaB}_{6(\mathrm{~s})}+6 \mathrm{CO}_{(\mathrm{g})} \leftrightarrow \mathrm{CaB}_{2} \mathrm{C}_{2(\mathrm{~s})}+2 \mathrm{~B}_{2} \mathrm{O}_{3(\mathrm{l})}+4 \mathrm{C}_{(\mathrm{s})} \\
& \mathrm{CaB}_{2} \mathrm{C}_{2(\mathrm{~s})}+3 \mathrm{CO}_{(\mathrm{g})} \leftrightarrow \mathrm{CaC}_{2(\mathrm{~s})}+\mathrm{B}_{2} \mathrm{O}_{3(\mathrm{l})}+3 \mathrm{C}_{(\mathrm{s})}
\end{aligned}
$$

A resistência a oxidação dos tijolos refratários de $\mathrm{MgO}$ $\mathrm{C}$ contendo $\mathrm{CaB}_{6}$ juntamente com $\mathrm{Al}$ ou ligas Al-Mg é melhorada devido a formação de uma camada de baixa porosidade encontrada na superfície do material. Esta camada será constituída principalmente por $3 \mathrm{MgO} \cdot \mathrm{B}_{2} \mathrm{O}_{3}$ formado a partir da reação do $\mathrm{MgO}$ com $\mathrm{B}_{2} \mathrm{O}_{3}$ (equação AA) [36].

O principal benefício da adição combinada de mais de um tipo de antioxidante é que ela inibe a oxidação do carbono e em alguns casos contribui para melhorar a resistência a corrosão e ao choque térmico dos refratários, porém ainda são necessários estudos mais conclusivos para especificar os mecanismos de atuação destes materiais.

\section{Outros antioxidantes}

O papel dos nitretos como $\mathrm{Si}_{3} \mathrm{~N}_{4}, \mathrm{BN}$ e $\mathrm{SiAlON}$ em refratários de $\mathrm{MgO}-\mathrm{C}$ ainda é pouco estudado e poucos trabalhos foram realizados com refratários dos sistemas $\mathrm{Al}_{2} \mathrm{O}_{3}-\mathrm{C}$ e $\mathrm{ZrO}_{2}-\mathrm{C}$ [32]. Assim, faz necessário um estudo mais detalhado sobre a atuação destes materiais.

Óxidos refratários tais como $\mathrm{Al}_{2} \mathrm{O}_{3}$ e $\mathrm{ZrO}_{2}$ não são considerados como tendo um efeito na oxidação do carbono, uma vez que eles não são reduzidos em temperaturas relativamente baixas. Entretanto, foi verificado que estes óxidos apresentam uma influência, até certo ponto, na oxidação do carbono devido as suas propriedades eletrônicas. $\mathrm{O} \mathrm{Al}_{2} \mathrm{O}_{3}$ é um receptor de elétrons e quando este se encontra na presença de carbono, ele pode receber os elétrons e formar uma nova distribuição eletrônica que irá facilitar a formação de $\mathrm{CO}(\mathrm{g})$ e acelerar a oxidação do carbono. Por outro lado $\mathrm{TiO}_{2}, \mathrm{ZrO}_{2}$ e $\mathrm{MgO}$ são doadores de elétrons e quando na presença do carbono, estes óxidos doam seus elétrons e assim a sua oxidação é inibida devido a formação de uma nova distribuição eletrônica. O carbono amorfo possui um grande numero de defeitos e ligações $\mathrm{C}-\mathrm{C}$ mais fracas do que o grafite, assim a sua oxidação parece não ser afetada por óxidos refratários [15].

\section{TENDÊNCIAS PARA O FUTURO}

Sabe-se que não há um antioxidante válido para todos os materiais e todas as situações. Resultados recentes revelaram que a mistura de vários antioxidantes é uma solução válida e as misturas de antioxidantes metálicos e não-metálicos são as mais interessantes do ponto de vista prático [6, 36, 46, 49]. $\mathrm{O}$ ideal seria combinar aditivos que proporcionem proteção ao carbono em temperaturas menores, como a formação de 
um líquido que atue sob fortes condições redutoras abaixo de $1400{ }^{\circ} \mathrm{C}$ e um sólido que proporcione um melhor resultado em condições oxidantes acima de $1400{ }^{\circ} \mathrm{C}$. A fase líquida formada em temperaturas menores deve envolver $\mathrm{O}$ carbono, ser fluída o suficiente para selar os poros dos refratários e se tornar sólida quando em contato com a atmosfera oxidante. Porém, estudos mais detalhados são necessários para se chegar a resultados satisfatórios.

Cálculos termodinâmicos não são usados somente para prever o comportamento de reação dos antioxidantes a elevadas temperaturas, mas também podem ajudar a seleção de antioxidantes apropriados para refratários contendo carbono. A desvantagem está no fato de que eles não fornecem qualquer informação da morfologia microestrutural e distribuição de cada produto de reação formado, e assim não podem revelar o efeito de cada fase na microestrutura dos refratários em elevadas temperaturas $[17,22]$. Para o completo entendimento dos mecanismos de reação dos antioxidantes em elevadas temperaturas, ambas análises, termodinâmica e microestrutural, são necessárias. Consequentemente, o entendimento atual da seqüência de reação e evolução microestrutural está longe de ser completado.

Outra opção a ser estudada é o uso de antioxidantes com tamanho de partículas nanométricos. Alguns trabalhos recentes estudaram a adição do $\mathrm{TiO}_{2}$ nanométrico juntamente com partículas micrométricas de alumínio em refratários de $\mathrm{MgO}-\mathrm{C}[47,50]$. A adição de 0,4\%-peso de $\mathrm{TiO}_{2}$ com 2,4\%-peso de $\mathrm{Al}$ proporcionou um aumento considerável (aproximadamente maior que o dobro) dos valores do módulo de ruptura a frio e a quente dos tijolos de $\mathrm{MgO}-\mathrm{C}$, quando comparado com um material sem a adição de antioxidantes. Este resultado foi atribuído a formação de fases como $\mathrm{Al}_{4} \mathrm{C}_{3}$ e $\mathrm{TiCN}$ que se encontram uniformemente distribuídas ao longo da matriz do refratário e assim atuam como um reforço. Além disso, o $\mathrm{TiO}_{2}$ nanométrico em adição com o pó convencional de alumínio não somente conduz a um aumento na resistência a oxidação do carbono, mas também atua na obtenção de menores valores do módulo elástico e no aumento da resistência ao choque térmico dos refratários. Por outro lado, o uso individual de $\mathrm{TiO}_{2}$ nanométrico pode agir negativamente nas propriedades mecânicas do tijolo refratário, sendo portanto tal procedimento não recomendado [50]. A atuação de diferentes antioxidantes nanométricos, sua produção, a definição do teor ideal destes materiais a serem adicionados e as possíveis contribuições destes às propriedades dos refratários são pontos importantes a serem estudados. Acredita-se que os antioxidantes nanométricos possam atuar efetivamente na prevenção da oxidação e na manutenção da integridade dos refratários contendo carbono.

\section{AGRADECIMENTOS}

Os autores agradecem à FAPESP pelo apoio recebido.

\section{REFERÊNCIAS}

[1] E. M. M. Ewais, "Carbon based refractories", Ceram. Soc. Japan 112, 10 (2004) 517-532.

[2] S. Zhang, W. Lee, "Carbon containing castables: current status and future prospects", Brit. Ceram. Trans. 101, 1 (2002) 1-8.

[3] Y. E. Pivinskii, O. G. Us'Yarov, "A new generation of unshaped refractories", Refract. Ind. Ceramic. 47, 1 (2006) 30-35.

[4] J. N. Niu, S. Terayama, T. Kiminami, N. Muroi, "Effect of graphite grains on properties of castables for a blast furnace trough", J. Techn. Ass. Refract. 22, 3 (2002) 219 222.

[5] I. R. Oliveira, A. R. Studart, F. A. Silva Jr., V. C. Pandolfelli, "Artigo revisão: estabilização de suspensões aquosas contendo grafite”, Cerâmica 46, 300 (2000) 186195.

[6] M. Rigaud, New additives in carbon-bonded refractories. Ceramic: charting the future, Techna Srl. (1995) 399.

[7] X. Li, M. Rigaud, S. Palco, "Oxidation kinects of graphite phase in magnesia-carbon refractories", J. Am. Ceram. Soc. 78, 4 (1995) 965-971.

[8] S. K. Sadrnezhaad, S. Mahshid, B. Hashemi, Z. A. Nemati, "Oxidation mechanism of $\mathrm{C}$ in $\mathrm{MgO}-\mathrm{C}$ refractory bricks", J. Am. Ceram. Soc. 89, 4 (2006) 1308-1316, doi: 10.1111/j.1551-2916.2005.00863.x.

[9] M. A. Faghihi-Sani, A. Yamaguchi, "Effect of Al and alumina additions on oxidation rate of $\mathrm{mgO}-\mathrm{C}$ refractory", J. Ceram. Soc. Jap. 110, 8 (2002) 699-702.

[10] S. K. Sadrnezhaad, Z. A. Nemati, S. Mahshid, S. Hosseini, B. Hashemi, "Effect of Al antioxidant on the rate of oxidation of carbon in $\mathrm{MgO}-\mathrm{C}$ refractory". J. Am. Ceram. Soc. 90, 2 (2007) 509-515.

[11] O. S. Ozgen, B. Rand, "Kinects of oxidation of the graphite phase in alumina/graphite materials I - Effect of temperature and initial pore structure at a fixed graphite content", Brit. Ceram. Trans. J. 84 (1985) 70-76.

[12] O. S. Ozgen, B. Rand, "Kinects of oxidation of the graphite phase in alumina/graphite materials II - Materials with different graphite content, graphite flakes size and with clay or carbon bonds", Brit. Ceram. Trans. J. 84 (1985) 213-218.

[13] C. F. Cooper, I. C. Alexander, C. J. Hampson, "The role of graphite in the thermal shock resistance of refractories", Brit. Ceram. Trans. J. 84 (1985) 57-62.

[14] M. Guo, S. Parada, P. T. Jones, J. Van Dyck, E. Boydens, D. Durinck, B. Blanpain, P. Wollants, "Degradation mechanisms of magnesia-carbon refractories by highalumina stainless steel slags under vacuum", Ceram. Int. (2006), doi:10.1016/j.ceramint.2006.03.009.

[15] A. Yamaguchi, S. Zhang, J. Yu, "Effect of refractory oxides on the oxidation of graphite and amorphous carbon", J. Am. Ceram. Soc. 79, 9 (1996) 2509-2511.

[16] M. Chen, N. Wang, J. Yu, A. Yamaguchi, "Oxidation protection of $\mathrm{CaO}-\mathrm{ZrO}_{2}-\mathrm{C}$ refractories by addition of $\mathrm{SiC}$ ", Ceram. Int. (2006), doi:10.1016/j.ceramint.2006.07.004. 
[17] S. Zhang, W. E. Lee, "Influence of additives on corrosion resistance and corroded microstructures of $\mathrm{MgO}-$ C refractories", J. Eur. Ceram. Soc. 21 (2001) 2393-2405. [18] C. G. Aneziris, J. Hubalkova, R. Barabás, "Microstructure evaluation of $\mathrm{MgO}-\mathrm{C}$ refractories with $\mathrm{TiO}_{2}$ - and Al- additions", J. Eur. Ceram. Soc. 27 (2007) 73-78.

[19] T. R. Lipinski, R. Fichtner, T. Benecke, "Study of the oxidation protection of $\mathrm{MgO}-\mathrm{C}$ refractories by means of boron carbide", Steel Res. 63, 11 (1992) 493-495.

[20] A. S. Gokce, C. Gurcan, S. Ozgen, S. Aydin, "The effect of antioxidants on the oxidation behaviour of magnesia-carbon refractory bricks", Ceram. Int. (2006), doi:10.1016/j.ceramint.2006.10.004.

[21] S. Uchida, K. Ichikawa, "High-temperature properties of unburned MgO-C bricks containing Al and Si Powders", J. Am. Ceram. Soc. 81, 11 (1998) 2910-2916.

[22] S. Zhang, N. J. Marriott, W. E. Lee, “Thermochemistry and microstructures of $\mathrm{MgO}-\mathrm{C}$ refractories containing various antioxidants", J. Eur. Ceram. Soc. 21 (2001) 10371047.

[23] A. R. Studart, M. D. M. Innocentini, I. R. Oliveira, V. C. Pandolfelli, "Reaction of aluminum powder with water in cement-containing refractory castables", J. Eur. Ceram. Soc. 25 (2005) 3135-3143.

[24] V. G. Domiciano, J. R. Garcia, V. C. Pandolfelli, "Resistência a corrosão de pós metálico sem concretos refratários contendo elevado teor de carbono", Cerâmica 52, 323 (2006) 136-142.

[25] I. R. Oliveira, A. R. Studart, B. A. Menegazzo, V. C. Pandolfelli, "Dispersão e comportamento reológico de concretos refratários ultra-baixo teor de cimento no sistema $\mathrm{Al}_{2} \mathrm{O}_{3}-\mathrm{SiC}-\mathrm{SiO}_{2}-\mathrm{C}$ ", Cerâmica 49, 309 (2003) 17-22.

[26] V. Domiciano, J. R. Garcia, V. C. Pandolfelli, "Water corrosion resistance of metal powders for carbon containing castables", Am. Ceram. Soc. Bull. 86 (2007) 9401-9406.

[27] V. Domiciano, J. R. Garcia, V. C. Pandolfelli, "Hydration resistance of silicon powder in high-carboncontaining refractory castables", Am. Ceram. Soc. Bull. 84, 10 (2005) 31-36.

[28] V. Domiciano, I. R. Oliveira, V. C. Pandolfelli, "Tendência à hidratação do pó de alumínio em concretos refratários contendo elevado teor de carbono", Cerâmica 50, 315 (2004) 225-230.

[29] V. Domiciano, I. R. Oliveira, R. Salomão, V. C. Pandolfelli, "Hydration of aluminum powder in highcarbon-containing refractory castables", Am. Ceram. Soc. Bull. 84, 5 (2005) 9101-9105.

[30] M. Innocentini, L. Nascimento, A. E. M. Paiva, V. C. Pandolfelli, B. Menegazzo, L. R. Bittencourt, "Aluminumcontaining refractory castables - Part I, evaluation of hydrogen-gas generation", Am. Ceram. Soc. Bull. 82, 6 (2003) 45-51.

[31] M. N. Khezrabadi, J. Javadpour, H. R. Rezaie, R. Naghizadeh, "The effect of additives on the properties and microstructures of $\mathrm{Al}_{2} \mathrm{O}_{3}-\mathrm{C}$ refractories", J. Mater. Sci. 41, 10 (2006) 3027-3032.
[32] C. Chan, B. B. Argent, W. E. Lee, "Influence of additives on slag resistance of $\mathrm{Al}_{2} \mathrm{O}_{3}-\mathrm{SiO}_{2}-\mathrm{SiC}-\mathrm{C}$ refractory bond phases under reducing atmosphere", J. Am. Ceram. Soc. 81, 12 (1998) 3177-3188.

[33] C. F. Chan, B. B. Argent, W. E. Lee, "Prediction of the effect additives on slag resistance of $\mathrm{Al}_{2} \mathrm{O}_{3}-\mathrm{SiO}_{2}-\mathrm{SiC}-\mathrm{C}$ bond phases in air", Calphad 27, 1 (2003) 115-125.

[34] K. Ichikawa, H. Nishio, O. Nomura, Y. Hoshiyama, "Suppression effects of aluminum on oxidation of MgO-C bricks", Taikabutsu Overseas 15, 2 (1995) 21-24.

[35] S. K. Nandy, N. K. Ghosh, D. Ghosh, G. C. Das, "Hydration of coked MgO-C-Al refractories", Ceramic. Int. 32, (2006) 163-172.

[36] S. Hanagiri, T. Harada, S. Aso, S. Fujihara, H. Yasui, S. Takanaga, H. Takahashi, A. Watanabe, "Effects of the addition of metal and $\mathrm{CaB}_{6}$ to magnesia carbon bricks for converters", Taikabutsu Overseas 13, 3 (1993) 20-27.

[37] Y. W. Li, C. G. Aneziris, X. X. Yi, S. L. Jin, N. Li, "Formation of dumbbell-shaped $\square$-SiC whiskers in $\mathrm{Al}_{2} \mathrm{O}_{3}$ $\mathrm{ZrO}_{2}-\mathrm{C}$ composite refractories", Interceram (2005) 20-23.

[38] M. N. Khezrabadi, J. Javadpour, H. R. Rezaie, R. Naghizadeh, "The effect of additives on the properties and microstructures of $\mathrm{Al}_{2} \mathrm{O}_{3}-\mathrm{C}$ refractories”, J. Mater. Sci. 41 (2006) 3027-3032.

[39] M. Skalska, W. Zelik, T. R. Lipinski, J. Greim, C. Klopfer, "Application of a new generation of antioxidants in carbon-containing bricks. Trials in the steel industry", In $49^{\text {th }}$ International Colloquium on Refractories, Aachen (2006) 117-120.

[40] S. Hayashi, S. Takanaga, H. Takahashi, A. Watanabe, "Behavior of boric compounds added in MgO-C bricks", Taikabutsu Overseas 11, 3 (1991) 12-19.

[41] T. Wang, A. Yamaguchi, "Synthesis of $\mathrm{Al}_{8} \mathrm{~B}_{4} \mathrm{C}_{7}$ and its oxidation properties in air", J. Ceram. Soc. Japan 108, 4 (2000) 375-380.

[42] A. Yamaguchi, S. Zhang, "Synthesis and some properties of $\mathrm{Al}_{4} \mathrm{SiC}_{4}$ ", J. Ceram. Soc. Japan 103, 1 (1995) 20-24.

[43] T. Wang, A. Yamaguchi, "Oxidation protection of $\mathrm{MgO}-\mathrm{C}$ refractories by means of $\mathrm{Al}_{8} \mathrm{~B}_{4} \mathrm{C}_{7}$ ”, J. Am. Ceram. Soc. 84, 3 (2001) 577-582.

[44] T. Wang, A. Yamaguchi, "Antioxidation behavior and effect of $\mathrm{Al}_{8} \mathrm{~B}_{4} \mathrm{C}_{7}$ added to carbon-containing refractories", J. Ceram. Soc. Japan 108, 9 (2000) 818-822.

[45] S. Zhang, A. Yamaguchi, "Effect of $\mathrm{Al}_{4} \mathrm{SiC}_{4}$ addition to carbon-containing refractories", J. Ceram. Soc. Japan 103, 3 (1995) 235-239.

[46] A. Yamaguchi, H. Tanaka, "Behavior and effects of $\mathrm{ZrB}_{2}$ added to carbon-containing refractories", Taikabutsu Overseas 15, 2 (1995) 3-9.

[47] C. G. Aneziris, U. Klippel, "Thermal shock behaviour of carbon bonded $\mathrm{MgO}-\mathrm{C}$ refractories with inorganic micro- and/or nano additions", In $5^{\text {th }}$ European Oxygen Steelmaking Conf., Aachen (2006) 1-6.

[48] T. Suruga, "Effect of Mg-B material addition to MgOC bricks", Taikabutsu Overseas 15, 2 (1995) 25-31.

[49] K. Ichikawa, N. Tsukamoto, O. Nomura, N. Imai, 
"Effects of Mg-B material addition to $\mathrm{Al}_{2} \mathrm{O}_{3}-\mathrm{C} \mathrm{SV}$ plate", Taikabutsu Overseas 15, 2 (1995) 33-37.

[50] U. Klippel, C. G. Aneziris, "Prospects of ceramic nanoparticles as additives for carbon-bonded $\mathrm{MgO}-\mathrm{C}$ refractories", In $5^{\text {th }}$ European Oxygen Steelmaking Conf., Aachen (2006) 6-9.

(Rec. 18/04/2007, Ac. 15/06/2007) 